\section{Drug-induced protein degradation heats up}

Two more companies have launched into the red-hot targeted protein degrader space. This trendy modality opens up new therapeutic avenues by targeting specific proteins for degradation, rather than for classical protein inhibition. Typically target degradation agents consist of bifunctional small molecules that bind a target with one arm and an E3 ubiquitin ligase with another, resulting in the target being tagged for destruction by the proteasome.

The newest entrants have disclosed few details of their approaches, as yet. Orionis Biosciences, which broke public ground in March, has been operating in stealth mode since 2015. One of the platforms they have developed is their Allo-Glues, small molecules that bind targets at allosteric sites to alter their form and function. At launch, Orionis disclosed that it is partnering with Novartis in a four-year deal to use this platform to find protein degraders and other drugs across therapeutic areas.

Amphista Therapeutics made its debut in April with the completion of a \$7.5-million series A funding round. Amphista's platform operates "independent of traditional E3 ubiquitin ligases," the company has said. It is on the hunt for cancer drugs.

More established targeted protein degrader players are also continuing to attract attention, as well as funding. Kymera Therapeutics closed on another $\$ 102$ million in March and plans to advance candidates into the clinic by next year. Nurix Therapeutics also raised another \$120 million in March and expects to file an Investigational New Drug application with the US Food and Drug Administration by the end of the year.

Last year Arvinas became the first company to advance a targeted protein degrader into the clinic. Yale University's Craig Crews, who kick-started the targeted degrader field with his seminal work on PROTACs (proteolysis-targeting chimeras), founded Arvinas in 2013. Before the COVID-19 shutdown, Arvinas had expected phase 1 dose-escalation data for their first-in-modality program in the second quarter of 2020 .

Published online: 11 May 2020 https://doi.org/10.1038/s41587-020-0529-9 labs, while also eliminating the inherent variability that arises even with skilled and experienced bench scientists. LabGenius's Field says that "these platforms change what it means to be a scientist ... with the object of maximizing time spent doing creative thinking and minimizing time spent executing in the lab."

This process can help researchers optimize multiple aspects of the drug development process in parallel - for example, simultaneously selecting compounds with optimal pharmacokinetic and toxicological properties as well as selective and strong on-target therapeutic effects. Many companies in this space are initially going after difficult clinical targets, including complex multifactorial diseases like inflammatory bowel disease or cancer, where the root causes may be poorly understood, and may which require carefully calibrated treatment with multiple drugs. "I believe that with our approach, we can really tackle many different networks that affect the behavior of the cell," says Cellarity president Cristina Rondinone. She also notes that this approach could put drug companies in a position to engage in discovery science in the realm of disease research - an area that is still chiefly the domain of academia.

It is neither cheap nor straightforward to build strong capabilities in both wet lab experimentation and computational biology in parallel. On the one hand, the core techniques for machine learning are well established, and the broad availability of cloud computing has largely eliminated the need for massive investments in computational infrastructure. But, on the other hand, Koller points out that the scale and complexity of these data can quickly overwhelm conventional analytical pipelines. "We had to rewrite the machine learning algorithms because they're built for microscope images of 256 by 256 pixels, and ours are 20,000 by 80,000 ," she says. "And when you think about the multimodal nature of the models that we have to build, very few machine-learning tasks involve that level of challenge."

Team building can be an even tougher task. Some of the laboratory techniques being employed, such as single-cell RNA-seq, are sufficiently cutting-edge that it can be hard to find researchers with deep experience. And, on the computational side, companies are not just looking for data scientists, coders and engineers with the skills needed to push the limits of machine learning capabilities, but also competing with the deep pockets of tech titans like Google. "Are pharma companies really prepared to have a head of deep learning who is earning more than their head of drug discovery?" asks Crane. A culture of collaboration is also essential, lest these ambitious startups lapse into the siloed organizational structures that have limited the impact of machine learning in drug development in the past.

But this work and investment could ultimately pay off massively if it succeeds in improving the efficiency - and thus reducing the cost - of drug development. Koller thinks the benefit is clearly worth the risk. "The front end is the wrong place to save," she says. "If a drug discovery program costs well over $\$ 1$ billion, you don't want to save \$10-15 million on a front-end pipeline that would create the right dataset and make the right predictions." The first products of this approach are now starting to trickle into the clinical pipeline, most notably a drug candidate for obsessive-compulsive disorder developed by Exscientia for Sumitomo Dainippon Pharma, which entered phase 1 testing earlier this year. However, it remains unclear what impact this development process will have on success rates in the clinic - particularly in the later phases of efficacy testing, where seemingly promising drug candidates routinely falter. "We are working at the edge of chaos, where we're interfering with living, adaptive systems," cautions Schneider. "It would be wrong to hope for perfect predictions."

Nevertheless, a growing number of major industry players are proving eager to explore this approach. For example, Celsius is now working with Janssen on identifying biomarkers of response to a combination therapy for ulcerative colitis, and Insitro is gearing up to present preliminary data from a collaboration with Gilead to devise new drugs for the notoriously difficult-to-treat liver disorder nonalcoholic steatohepatitis. But these startups are not merely looking to act as collaborative service providers. "At our core, we are a precision medicine company, and our long-term goal is to develop and commercialize therapies independently," says Celsius CEO Tariq Kassum.

Indeed, Hopkins sees this approach as the natural next evolutionary step for the industry. He cites the example of chess grandmaster Garry Kasparov, who, after being bested by IBM's Deep Blue computer, teamed up with the AI to achieve a gameplay style more formidable than either man or machine alone. "This is not about replacing the drug designer," he says. "This is about enhancing their abilities and increasing their probability of getting a drug into clinical trials."

Michael Eisenstein

Philadelphia, PA, USA

Published online: 11 May 2020

https://doi.org/10.1038/s41587-020-0521-4 